\title{
A Short Note on Nonlocal Transformations and First Integrals for Certain Nonlinear Oscillator Equations
}

\author{
Emrullah Yaşar \\ Uludag University, Faculty of Arts and Sciences, Department of Mathematics, P.O. Box 16059, Görükle, Bursa, Turkey \\ Correspondence should be addressed to Emrullah Yaşar; eyasar@uludag.edu.tr \\ Received 18 February 2013; Revised 18 May 2013; Accepted 20 May 2013 \\ Academic Editor: Patricia J. Y. Wong \\ Copyright (c) 2013 Emrullah Yaşar. This is an open access article distributed under the Creative Commons Attribution License, \\ which permits unrestricted use, distribution, and reproduction in any medium, provided the original work is properly cited.

\begin{abstract}
This short note presents a simple and effective application of the generalized Sundman transformations and the Jacobi last multiplier approach for two nonlinear oscillator equations. We reobtain the first integrals with this method very easily. In addition, we also compute the Sundman symmetries and Lagrangians.
\end{abstract}

\section{Introduction and Preliminaries}

The problem of integrability of ordinary differential equations (ODEs) has been extensively studied. In [1], Guha et al. proposed the generalized Sundman transformation (GST) method for second-order nonlinear ODEs. With this method, one can obtain easily the first integrals of the given equation. Another alternative method is the Jacobi last multiplier (JLM) approach [2]. In this approach, one can construct the Lagrangian of the given equation. Then using this Lagrangian via Noether's theorem, one finds the first integrals of the given equation. We applied both methods to the following two nonlinear oscillator equations:

$$
\begin{gathered}
\ddot{x}-\frac{k x \dot{x}^{2}}{\left(1+k x^{2}\right)}+\frac{\alpha^{2} x}{\left(1+k x^{2}\right)}=0, \\
\ddot{x}+\frac{k x \dot{x}^{2}}{\left(1+k x^{2}\right)}+\frac{\alpha^{2} x}{\left(1+k x^{2}\right)^{3}}=0,
\end{gathered}
$$

where $k$ and $\alpha$ are arbitrary parameters. Bruzon et al. [3]. studied and obtained first integrals of the previous equations by the nonlocal symmetry method. In this work, we reobtain these first integrals without much more efforts by the methods of $[1,2]$.

Now, we present notation to be used and recall the theoretical backgrounds that appear in $[1,2]$.
The Jacobi equation is given by

$$
\ddot{x}+\frac{1}{2} \phi_{x} \dot{x}^{2}+\phi_{t} \dot{x}+B(t, x)=0
$$

and may be transformed to $X^{\prime \prime}=0$ under the GST

$$
X(T)=F(t, x), \quad d T=G(t, x) d t, \quad \frac{\partial F}{\partial x} \neq 0, \quad G \neq 0 .
$$

The important subclass of (3) is $\phi_{t}=0=B_{t}$, namely,

$$
\ddot{x}+\frac{1}{2} \phi_{x} \dot{x}^{2}+B(x)=0 .
$$

There are a number of equations which belong to this subclass. In this case, (5) is mapped to the equation

$$
X^{\prime \prime}+X=0
$$

where $X^{\prime}=d X / d T$. The form of GST which maps (5) to (6) is as follows

$$
\begin{gathered}
X=F(x)=\left( \pm \frac{2}{\beta^{2}} \int B(x) \exp \phi(x) d x\right)^{1 / 2}, \\
d T=\frac{B(x) \exp (\phi(x) / 2)}{\left( \pm \int B(x) \exp \phi(x) d x\right)^{1 / 2}} d t
\end{gathered}
$$


where $\beta$ is a constant. The latter is obviously a nonlocal transformation. Note that a first integral for (6) is obviously

$$
X^{\prime 2}+X^{2}=I_{1} .
$$

It is well known that a Sundman symmetry for (3) is a transformation of the form

$$
\begin{aligned}
& F(\widetilde{t}, \tilde{x})=M(F(t, x), G(t, x)), \\
& G(\widetilde{t}, \tilde{x}) d \widetilde{t}=N(F(t, x), G(t, x)) d t,
\end{aligned}
$$

where $M$ and $N$ are some differentiable functions such that the transformation keeps (3) invariant. In other words, (3) is transformed to

$$
\ddot{\tilde{x}}+\frac{1}{2} \phi_{\tilde{x}} \dot{\tilde{x}}^{2}+\phi_{\dot{t}} \dot{\tilde{x}}+B(\widetilde{t}, \tilde{x})=0 .
$$

Therefore, we have the following Sundman symmetry for (5):

$$
\begin{aligned}
& F(\tilde{t}, \tilde{x})= \pm \sqrt{F^{2}(t, x)+c}, \\
& G(\tilde{t}, \tilde{x}) d \widetilde{t}= \pm G(t, x) \frac{F}{\sqrt{F^{2}+c}} d t .
\end{aligned}
$$

Now, we recall the JLM and Lagrangian for second-order ODEs. Let us consider the following class of system:

$$
\begin{gathered}
\dot{x}=p_{1}(x) y, \\
\dot{y}=q_{0}(x)+q_{2}(x) y^{2} .
\end{gathered}
$$

Such a system corresponds to

$$
\ddot{x}+f(x) \dot{x}^{2}+g(x)=0,
$$

with

$$
f(x)=-\left(\frac{p_{1}^{\prime}(x)+q_{2}(x)}{p_{1}(x)}\right), \quad g(x)=-p_{1}(x) q_{0}(x) .
$$

The JLM for (5) is given by

$$
M(x)=\left(\exp \int^{x} f(s) d s\right)^{2} .
$$

It is necessary to point out that for (5) the JLM is independent of $\dot{x}$. It is well known that the relationship between Lagrangian and the JLM has the following identity:

$$
M=\frac{\partial^{2} L}{\partial \dot{x}^{2}}
$$

Using (17) and (16), the Lagrangian of (5) has the form of

$$
L(x, \dot{x})=M(x) \frac{\dot{x}^{2}}{2}+\int^{x} M(s) q_{0}(s) p_{1}(s) d s .
$$

\section{Main Results}

Firstly, we consider the nonlinear oscillator equation (1). Comparison with the Jacobi equation (5) reveals that

$$
\frac{1}{2} \phi_{x}=-\frac{k x}{\left(1+k x^{2}\right)}, \quad \phi_{t}=0, \quad B(t, x)=\frac{\alpha^{2} x}{\left(1+k x^{2}\right)} .
$$

Hence, from (19), $\phi(x, t)=\ln \left(1+k x^{2}\right)^{-1}$. As a result from (8) taking the positive square root, we find $F(x)=$ $(i / \beta)\left(\alpha / \sqrt{k^{2} x^{2}+k}\right)$, and it turns out that $G=-(i \alpha \sqrt{k} x /(1+$ $\left.k x^{2}\right)$ ). Hence, the Sundman transformation has the explicit form

$$
X=F(x)=\frac{i}{\beta} \frac{\alpha}{\sqrt{k^{2} x^{2}+k}}, \quad d T=-\frac{i \alpha \sqrt{k} x}{\left(1+k x^{2}\right)} d t .
$$

We aim to obtain the first integral of (1) using the transformations (20). Calculating the first derivative of $X(T)$,

$$
\frac{d X}{d T}=\frac{1}{\beta}\left(1+k x^{2}\right)^{-1 / 2} \dot{x},
$$

and then substituting it to (9), we obtain the following first integral:

$$
I_{1}=\frac{\alpha^{2}-k \dot{x}^{2}}{1+k x^{2}} .
$$

We note that (22) has exactly the same result which appeared in [3].

To explore the first integral associated with nonlinear oscillator of (2), repeating the procedure previously given, we obtain the following Sundman transformation:

$$
X=F(x)=\frac{i}{\beta} \frac{\alpha}{\sqrt{k^{2} x^{2}+k}}, \quad d T=-\frac{i \alpha \sqrt{k} x}{\left(1+k x^{2}\right)^{2}} d t .
$$

Calculating the first derivative of $X(T)$ and then substituting it to (9), we obtain the following first integral:

$$
I_{2}=\left(k x^{2}+1\right) \dot{x}^{2}-\frac{\alpha^{2}}{k\left(1+k x^{2}\right)} .
$$

We note that (24) has exactly the same result which appeared in [3]. Now, we try to find the Sundman symmetries of (1) and (2). The Sundman symmetry for (1) being of the form (10), we use (12). Consequently with $F$ and $G$ given as in (20), one finds that

$$
\begin{gathered}
\tilde{x}=\left[\sqrt{\left.\frac{-\alpha^{2} x^{2}-c \beta^{2}\left(k x^{2}+1\right)}{-\alpha^{2}+c \beta^{2}\left(k^{2} x^{2}+k\right)}\right],}\right. \\
\tilde{t}=A+i \int \alpha\left[1+k\left(\frac{-\alpha^{2} x^{2}-c \beta^{2}\left(k x^{2}+1\right)}{-\alpha^{2}+c \beta^{2}\left(k^{2} x^{2}+k\right)}\right)\right] \\
\times \frac{x}{\left(1+k x^{2}\right) \sqrt{-\alpha^{2} x^{2}-c \beta^{2}\left(k x^{2}+1\right)}} d t
\end{gathered}
$$


where $A$ is a constant of integration. Repeating (12) for (2) with $F$ and $G$ given as in (23), one finds that

$$
\begin{gathered}
\tilde{x}=\left[\sqrt{\left.\frac{-\alpha^{2} x^{2}-c \beta^{2}\left(k x^{2}+1\right)}{-\alpha^{2}+c \beta^{2}\left(k^{2} x^{2}+k\right)}\right],}\right. \\
\widetilde{t}=A+i \int \alpha\left[1+k\left(\frac{-\alpha^{2} x^{2}-c \beta^{2}\left(k x^{2}+1\right)}{-\alpha^{2}+c \beta^{2}\left(k^{2} x^{2}+k\right)}\right)\right]^{2} \\
\times \frac{x}{\left(1+k x^{2}\right)^{2} \sqrt{-\alpha^{2} x^{2}-c \beta^{2}\left(k x^{2}+1\right)}} d t
\end{gathered}
$$

where $A$ is a constant of integration. Now, we try to find the Lagrangians of (1) and (2). Equation (1) can be considered as the following system:

$$
\dot{x}=y, \quad \dot{y}=\frac{k x y^{2}}{1+k x^{2}}-\frac{\alpha^{2} x}{1+k x^{2}} .
$$

Consequently from (14), we have $f(x)=-k x /\left(1+k x^{2}\right)$ and $g(x)=\alpha^{2} x /\left(1+k x^{2}\right)$. The JLM from (16) is given by $M(x)=1 /\left(1+k x^{2}\right)$, and the Lagrangian from (18) is

$$
L(x, \dot{x})=\frac{\dot{x}^{2}}{2\left(1+k x^{2}\right)}+\frac{\alpha^{2}}{2\left(k^{2} x^{2}+k\right)} .
$$

We note that (28) has exactly the same result which appeared in [4]. Repeating the procedure previously given, we find the following Lagrangian for (2):

$$
L(x, \dot{x})=\frac{1}{2}\left(1+k x^{2}\right) \dot{x}^{2}+\frac{\alpha^{2}}{2\left(k^{2} x^{2}+k\right)} .
$$

\section{References}

[1] P. Guha, B. Khanra, and A. G. Choudhury, "On generalized Sundman transformation method, first integrals, symmetries and solutions of equations of Painlevé-Gambier type," Nonlinear Analysis: Theory and Methods, vol. 72, no. 7-8, pp. 3247-3257, 2010.

[2] A. G. Choudhury and P. Guha, "On isochronous cases of the Cherkas system and Jacobi's last multiplier," Journal of Physics A, vol. 43, no. 12, Article ID 125202, 2010.

[3] M. S. Bruzon, M. L. Gandarias, and M. Senthilvelan, "On the nonlocal symmetries of certain nonlinear oscillators and their general solution," Physics Letters A, vol. 375, no. 33, pp. 29852987, 2011.

[4] Z. E. Musielak, "Standard and non-standard Lagrangians for dissipative dynamical systems with variable coefficients," Journal of Physics A, vol. 41, no. 5, Article ID 055205, 2008. 


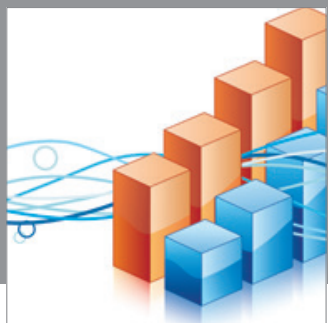

Advances in

Operations Research

mansans

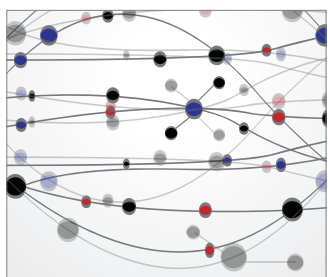

The Scientific World Journal
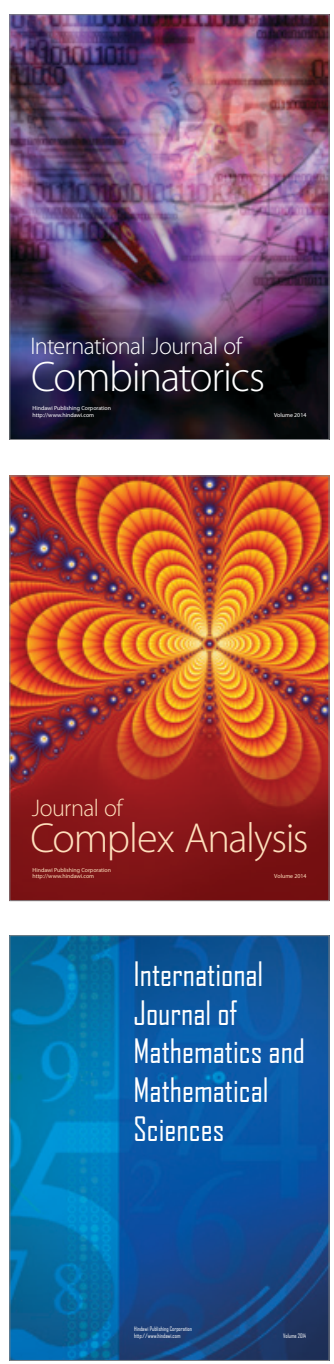
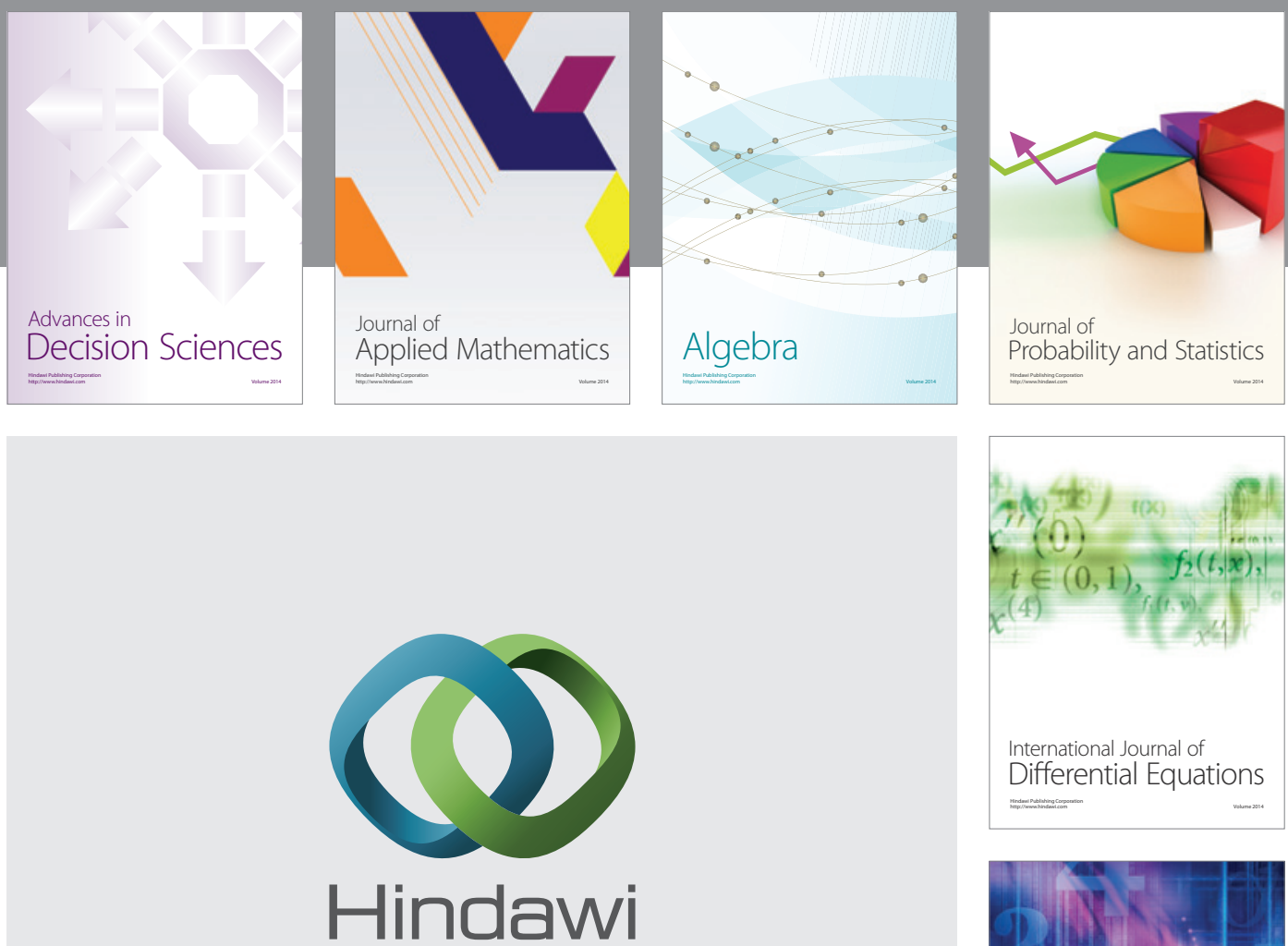

Submit your manuscripts at http://www.hindawi.com
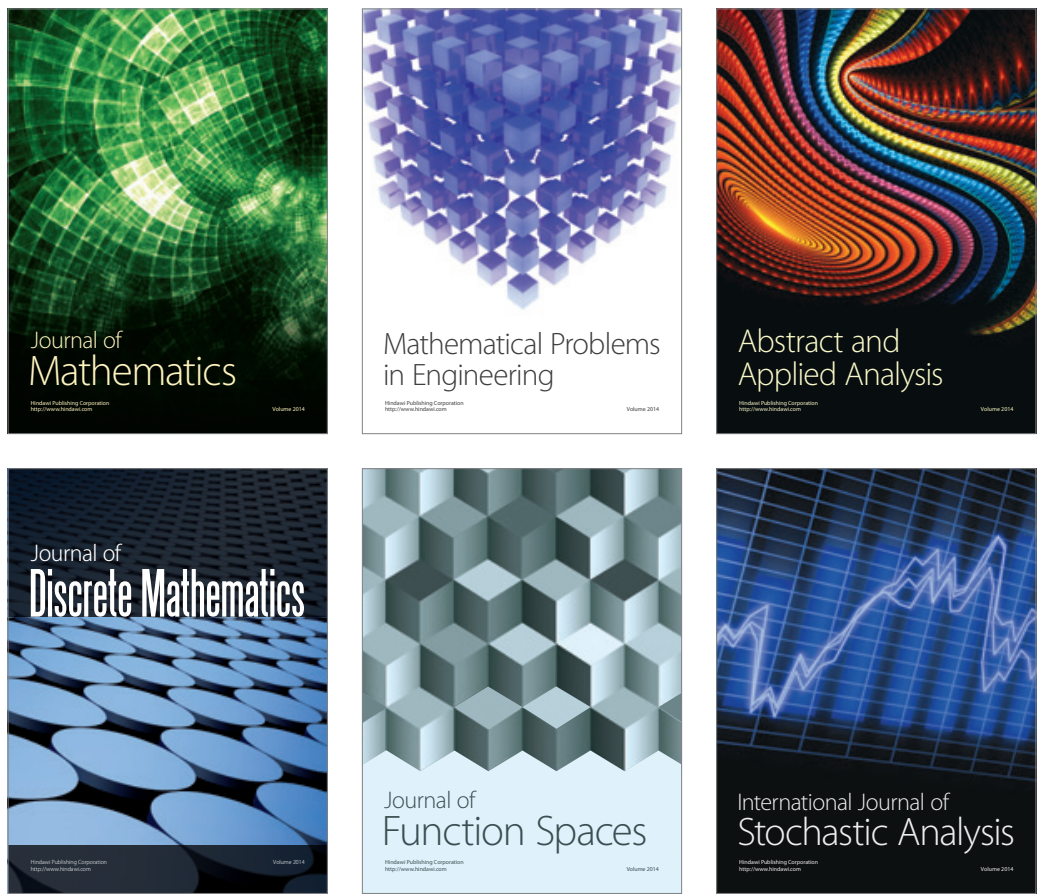

Journal of

Function Spaces

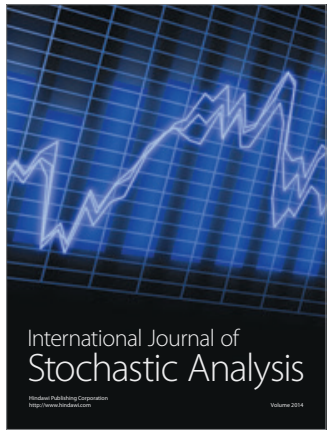

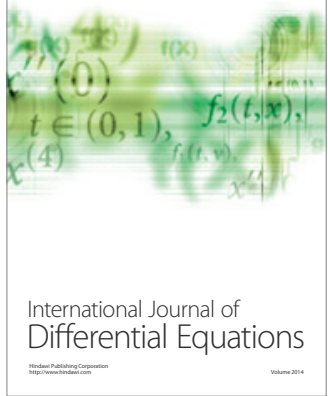
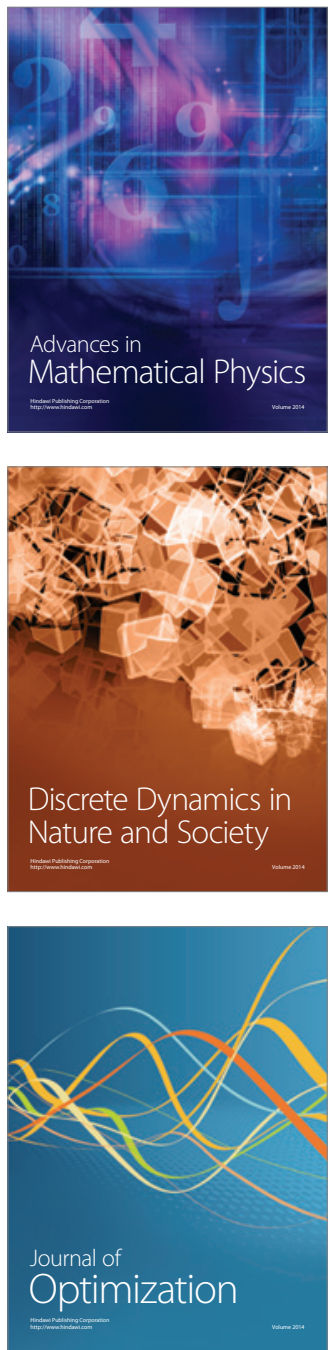